ARTICLE INFO

Received 20 March 2020

Accepted 26 March 2020

Available online April 2020

\title{
Fluorinated acrylic polymers with fluorine-containing electro-optical chromophores in side chain
}

\author{
V.I. Sokolov ${ }^{1.2}$, A.S. Akhmanov ${ }^{1.2}$, I.M. Asharchuk ${ }^{1}$, I.O. Goryachuk ${ }^{1}$, S.I. Molchanova ${ }^{1}$, \\ Yu.E. Pogodina ${ }^{3}$, E.V. Polunin ${ }^{3}$, K.V. Khaidukov ${ }^{1}$ \\ ${ }^{1}$ Institute of Photonic Technologies, Federal Scientific Research Center "Crystallography and \\ Photonics" RAS, Moscow, Russia \\ visokol@rambler.ru. \\ ${ }^{2}$ Federal Research Center "Scientific Research Institute for System Research” RAS, Moscow, \\ Russia \\ ${ }^{3}$ Institute of Organic Chemistry n.a. N.D. Zelinsky RAS, Moscow, Russia
}

\begin{abstract}
A new electro-optical polymer has been synthesized with a fluorine-containing electrooptical chromophore covalently connected to a fluorine-containing macromolecule. The degree of fluorination of polymer, defined as the ratio of the number of fluorine atoms to the number of fluorine and hydrogen atoms in this macromolecule, is $28.6 \%$. The optical and physical properties of the polymer obtained were investigated. It was shown that it is capable to film formation and can be used to create optical waveguides. It was found that under the action of laser radiation in visible range, the irreversible photobleaching of chromophore in side chain occurs, which is accompanied by decrease in the polymer refractive index. The single-mode channel waveguides in the electrooptical polymer were formed by photobleaching. The polymer possesses increased optical transparency in the telecommunications $\mathrm{C}$-wavelength range and is promising for creation of electro-optical modulators operating in the near IR range.
\end{abstract}

Keywords: fluorine-containing electro-optical chromophores, fluorine-containing acrylic polymers, photobleaching, laser drawing, polymer waveguides.

\section{Introduction}

Electro-optical (EO) polymeric materials are widely used for creation of high-speed integrated optical modulators and switches operating in telecommunications $\mathrm{C}$-wavelength range of 
1530-1565 nm [1-8]. To create such materials, the chromophores are used that can change the refractive index under the influence of an applied electric field. An electro-optical polymer can be made either by introducing the chromophore molecules into a passive matrix (guest-host system) [4-6], or by covalently incorporating them into side chains of polymer macromolecules (side-chain system) [7-9]. The latter approach is more promising, since it prevents the agglomeration of chromophores, resulting in decrease of electro-optical coefficient $r 33$. The significant importance is the synthesis of new EO-polymers, which, along with a large coefficient $r 33$, have high optical transparency in the C-range. An increase in transparency can be achieved by using fluorinecontaining chromophores and polymers, since the replacement of light hydrogen atoms with heavier fluorine atoms leads to a shift of vibrational absorption bands towards longer wavelengths [10]. As a result, the transparency windows of EO-polymer material are opened in C - region of spectrum. In addition, the replacement of hydrogen atoms with fluorine atoms can increase the electronegativity of acceptor part of chromophore molecule, increasing its dipole moment.

Formely it was reported about the creation of a number of fluorine-containing polymers in which either a chromophore or a polymer contained the fluorine atoms $[8,9,11]$. In this article the synthesis of a new EO-polymer of "side-chain" type based on chromophore of "dispersed red", in which both chromophore and polymer are fluorine-containing is described. It is a copolymer of methyl methacrylate with covalently attached fluorine-containing chromophore DR1218 (see below) and fluorine-containing acrylate. The light-guide films $0.5-4 \mu \mathrm{m}$ thick were made from the EO-polymer. It was shown that under the action of laser radiation in visible range $(440 \mathrm{~nm})$, this polymer is bleached due to photodegradation of the chromophore in the side chain. It was found that in this process the refractive index $n$ of polymer material decreases, and the change in $n$ can reach $\Delta n=0.04$. This makes it possible to produce channel waveguides, waveguide splitters and other elements of integrated optical devices in light-guide polymer films by means of selective laser photobleaching.

\section{Synthesis of fluorine-containing electro-optical polymer MMA-DR1218/FluoroAc}

The synthesis of fluorine-containing polymers with EO-chromophore in the side chain is carried out, as a rule, in three stages [1-3]. At the first stage, the chromophore is synthesized itself. This chromophore is then acylated with methacryloyl chloride (or with its analogue) to form the ester. The third stage is the radical copolymerization of obtained ester with methyl methacrylate, methyl acrylate or with their fluorine-containing analogues. In this way, for example, a polymer based on alpha fluoroacrylate with covalently attached DR1 chromophore was synthesized [11]. In 
this study, we first used the fluorine-containing chromophore DR1218, the ester of which was copolymerized with fluorine-containing acrylate $\mathrm{CH}_{2}=\mathrm{CH}-\mathrm{C}(\mathrm{O}) \mathrm{O}-\mathrm{CH}_{2}-\mathrm{CF}_{3}$. Thus, the electrooptical polymer including both fluorine-containing chromophore and fluorine-containing macromolecule was first created. The synthesis procedure is described in Section 5.

\section{Study of optical and physical properties of the electro-optical polymer}

The properties of obtained polymer were studied by IR Fourier spectroscopy (FTIR), refractometry, prism excitation of waveguide modes, wide-angle X-ray diffraction, thermogravimetry and differential scanning calorimetry. In Fig. 1 shows the transmission spectrum of 32- $\mu$ m thick MMA-DR1218/FluoroAc copolymer film on the $\mathrm{KBr}$ substrate, measured via Shimadzu 8400S FTIR spectrometer. As can be seen from Fig. 1, the main absorption bands of this copolymer lie in the region of long waves (below $4000 \mathrm{~cm}^{-1}$;) in telecommunication C-wavelength range $1530-1565 \mathrm{~nm}(6390-6535 \mathrm{~cm}-1)$ the absorption of this polymer is small.

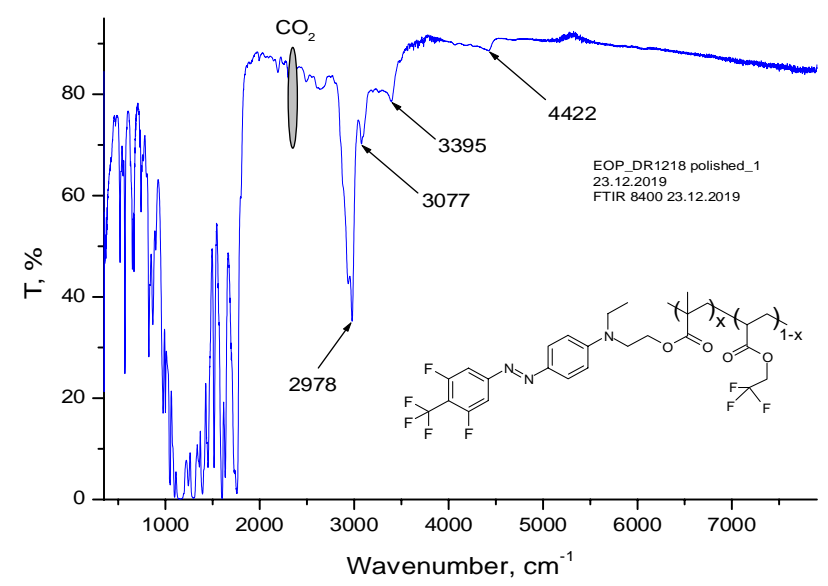

Figure 1. The transmission spectrum of MMA-DR1218/FluoroAc copolymer film on the $\mathrm{KBr}$ substrate, measured via Shimadzu 8400S FTIR spectrometer. The inset shows a fragment of polymer structure.

Fig. 2 shows the thermomechanical curve of polymer sample 3, measured via TMA Q400 instrument (TA Instruments, USA) at a heating rate of $5{ }^{\circ} \mathrm{C} / \mathrm{min}$ (with probe diameter $6 \mathrm{~mm}$ and force on the probe $1 \mathrm{~N}$ ). As follows from Fig. 2, the resulting material is characterized by two temperature transitions - the low temperature transition, which is associated with glass transition temperature, and high temperature transition, due to polymer flow. 


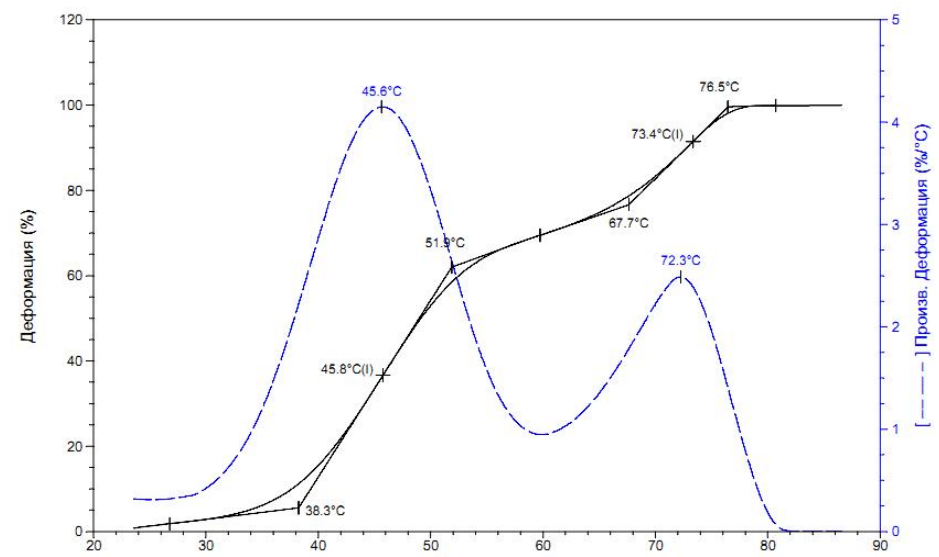

Figure 2. The thermomechanical curve of MMA-DR1218/FluoroAc polymer, measured via TMA Q400 instrument (TA Instruments, USA) at a heating rate of $5^{\circ} \mathrm{C} / \mathrm{min}$.

Low-temperature transition begins at $38.3{ }^{\circ} \mathrm{C}$ and ends at $51.9^{\circ} \mathrm{C}$, with the appearance of large deformation of this curve. Upon further heating, the sample passes into a fluid state with onset temperature of fluidity at $67.7^{\circ} \mathrm{C}$. Upon reaching $100 \%$ deformation at $76.5^{\circ} \mathrm{C}$, it was visually observed that liquid remained in a vial, which could indicate a linear structure of obtained polymer. The glass transition temperature Tg of synthesized polymer was determined in two ways: via three tangents or using the curve of strain derivative versus temperature. The found temperature of glass transition was $T_{\mathrm{g}} \approx 45.6{ }^{\circ} \mathrm{C}$.

The structure of EO-polymer was studied via Rigaku Miniflex600 wide-angle X-ray spectrometer $(\lambda=1.54178 \mathrm{~A})$. The diffractogram of MMA-DR1218/FluoroAc polymer film is shown in Fig. 3. It is seen that the spectrum of MMA-DR1218 / FluoroAc does not contain sharp diffraction peaks, while wide halo with centers near $2 \theta=18.8$ and $41.3 \mathrm{deg}$. are observed. From the analysis of diffraction pattern, we can conclude that the electro-optical MMA-DR1218/FluoroAc polymer is amorphous.

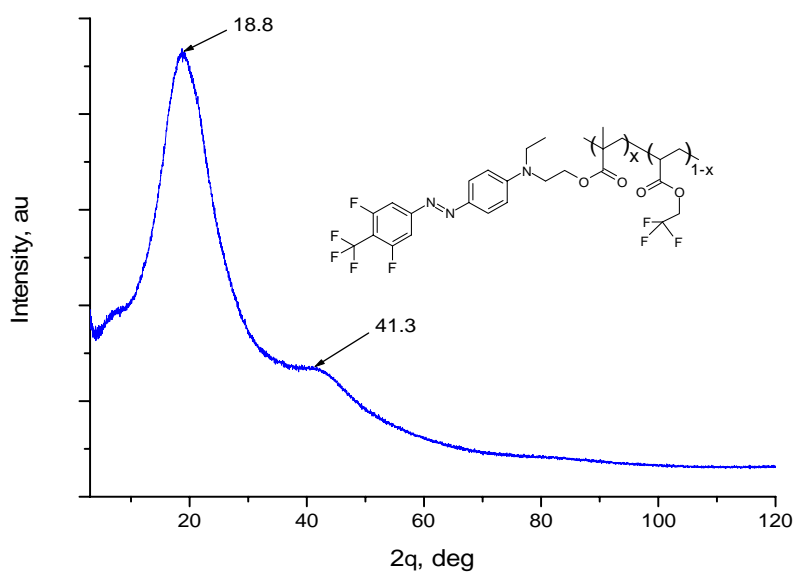


Figure 3. The diffraction pattern of MMA-DR1218/FluoroAc polymer film, measured via Rigaku Miniflex600 wide-angle $X$-ray spectrometer.

\section{Photobleaching of the electro-optical polymer}

Films made from synthesized EO-polymer were formed by centrifugation from solutions of MMA-DR1218/FluoroAc in chlorobenzene at the quartz substrates, as well as at the silicon substrates with a thermally growing oxide layer. The absorption spectrum of MMADR1218/FluoroAc film freshly applied to a quartz substrate is shown in Fig. 8 (Curve 1). As can be seen from Fig. 8, the polymer has the intense absorption band $103 \mathrm{~nm}$ wide centered near $438 \mathrm{~nm}$. Additional, less intense absorption band is located near $269 \mathrm{~nm}$. These bands are due to absorption of light by chromophore molecules. Note that electro-optical MMA-DR1218/FluoroAc polymer has a noticeable absorption only in visible and UV-regions of spectrum; in near IR-wavelength range, the absorption of this polymer is small (see Fig. 1 and Fig. 4).

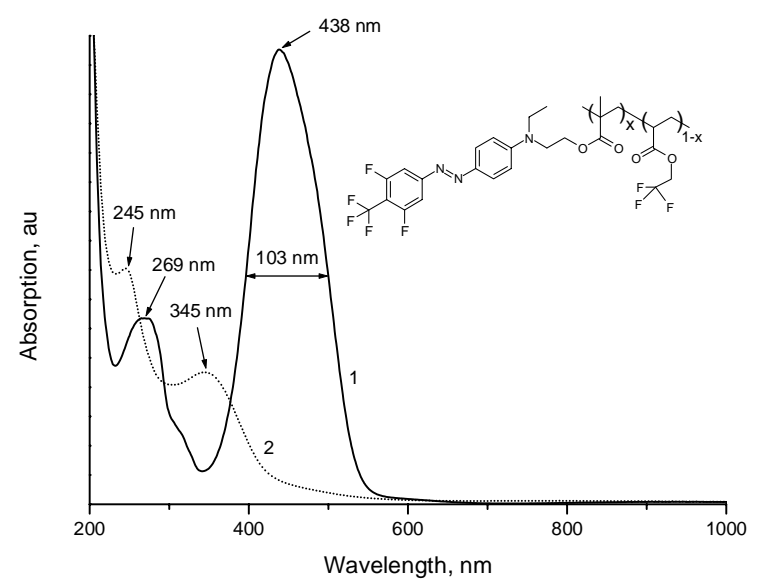

Figure 4. The absorption spectra of electro-optical MMA-DR1218/FluoroAc polymer film in visible and near IR-wavelength ranges.

(1) - before, (2) - after exposure by laser radiation with a wavelength at $440 \mathrm{~nm}$.

The inset shows the molecular structure of EO-polymer.

Fig. 4 also shows the absorption spectrum of this film after exposure by radiation from diode laser with intensity of $50 \mathrm{~W} / \mathrm{cm}^{2}$ and wavelength at $440 \mathrm{~nm}$, located near the absorption peak of MMA-DR1218/FluoroAc (Curve 2). A comparison of Curves 1 and 2 shows that during exposure, the intensity of absorption bands centered near 438 and $269 \mathrm{~nm}$ decreases; wherein the new absorption bands centered near 345 and $245 \mathrm{~nm}$ appear. Such a change in absorption spectrum is apparently due to irreversible photodegradation of chromophore, with loss of color (photobleaching effect $[6,12])$. 
The photodestruction of DR1218 chromophore molecules is accompanied by a change in their polarizability and, as a result, this change in refractive index $n$ of polymer material. To determine the magnitude of this change in refractive index $\Delta n$ by means of centrifugation on silicon substrates with a thermally growing oxide layer of $3.7 \mu \mathrm{m}$ thickness, the films of MMA-DR1218/FluoroAc polymer with a thickness of about $3.3 \mu \mathrm{m}$ were made. The polymer refractive index during photobleaching was measured by resonant excitation of waveguide modes in film at wavelength of $632.8 \mathrm{~nm}$ using Metricon/2010M prism coupling device (Metricon corp., USA). The measurement results are presented in Table. 1.

Table 1. Refractive indices $n$ of MMA-DR1218/FluoroAc copolymer film at wavelength of 632.8 $n$, measured via Metricon/2010M prism coupling device (with TE-and TM-polarization of probe laser beam).

\begin{tabular}{|c|c|c|}
\hline Polarization & $\begin{array}{c}\text { Refraction index } \boldsymbol{n} \text { of } \\
\text { freshly applied film }\end{array}$ & $\begin{array}{c}\text { Refraction index } \boldsymbol{n} \text { of film after exposure by radiation } \\
\text { from diode laser with wavelength of } \mathbf{4 4 0} \mathbf{~} \mathbf{m}\end{array}$ \\
\hline $\mathrm{TE}$ & 1.5823 & 1.5421 \\
\hline $\mathrm{TM}$ & 1.5824 & 1.5447 \\
\hline
\end{tabular}

From Tab. 1 it follows that refractive indices of a freshly applied polymer film for TE- and TM-polarization are close, which indicates its uniformity and isotropy. It is also seen that refractive index of polymer decreases during photobleaching, while this decrease can reach $\Delta n \approx 0.04$. Such change in $n$ is sufficient for the formation of channel optical waveguides with numerical aperture $\mathrm{NA}=0.35$. Thus, the electro-optical MMA-DR1218/FluoroAc polymer able to film formation with good quality.

\section{Laser drawing of waveguide structures in the EO-polymer}

Process of channel waveguides forming in films of electro-optical polymer [9, 12] proceeds as follows: under the influence of spatially selective photobleaching, the regions with a reduced refractive index in film are formed, serving as a waveguide sheath, and unexposed material with a higher $n$ is a light guide core in which optical radiation may propagate. Fig. 5 shows the photograph of waveguide splitter formed in MMA-DR1218 / FluoroAc film under the action of laser radiation at wavelength of $440 \mathrm{~nm}$. 


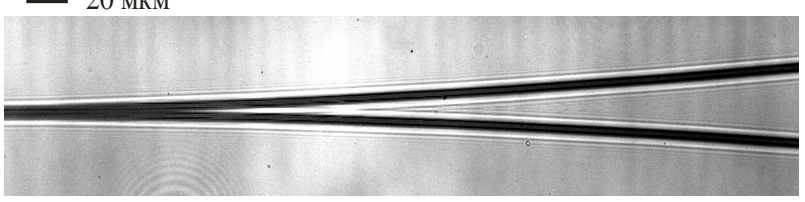

Figure 5. The photograph of waveguide splitter formed in MMA-DR1218/FluoroAc polymer film under the action of laser radiation at wavelength of $440 \mathrm{~nm}$. The dark stripes with a width of $10 \mu \mathrm{m}$ (unbleashed areas) are the light guide veins of waveguides.

Thus, by laser photobleaching of electro-optical polymer films, it is possible to produce various elements of integrated-optical devices, in particular, the waveguides and waveguide splitters, which are components of Mach-Zehnder interferometers and are necessary for creating high-speed integrated-optical modulators [13].

\section{Experimental part}

\subsection{Synthesis of fluorine-containing chromophore DR1218}

The synthesis scheme for EO-chromophore of 4-(4-trifluoromethyl-3,5-difluorophenylazo) N-ethyl-N- (2-hydroxyethyl) aniline DR1218 is shown in Fig. 6.

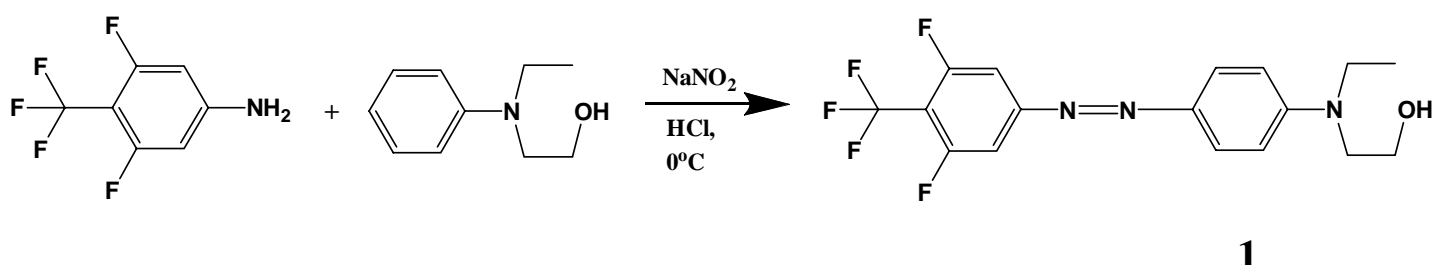

Figure 6. The synthesis scheme for EO-chromophore of 4-(4-trifluoromethyl-3,5difluorophenylazo)-N-ethyl-N-(2-hydroxyethyl) aniline (1) DR1218.

4-Trifluoromethyl-3,5-difluoroaniline $(6.122 \mathrm{~g}, 31 \mathrm{mmol})$ was dissolved in a mixture of $16.4 \mathrm{ml}$ concentraced $\mathrm{HCl}$ and $9.6 \mathrm{ml}$ of water, then cooled to $-3{ }^{\circ} \mathrm{C}$ and $2.14 \mathrm{~g}(31 \mathrm{mmol})$ of $\mathrm{NaNO}_{2}$ added in small portions over 1 hour. This solution was stirred for $30 \mathrm{~min}$ at a temperature of $-3-0{ }^{\circ} \mathrm{C}$. N-ethyl-N-hydroxyethylaniline $(4.9 \mathrm{ml}, 31 \mathrm{mmol}$ ) was dissolved in $15 \mathrm{ml}$ of $20 \% \mathrm{HCl}$; over 10 min this solution was added dropwise to reaction mixture and stirred at $0{ }^{\circ} \mathrm{C}$ for one hour. Then, $10 \% \mathrm{NaOH}$ solution was added at $10-20{ }^{\circ} \mathrm{C}$ before receiving $\mathrm{pH} 8$; this mixture was extracted $(3 \times 50 \mathrm{ml})$ with chloroform. The organic fraction was dried over anhydrous $\mathrm{Na}_{2} \mathrm{SO}_{4}$, filtered and evaporated in vacuo to constant weight. Received $9.2 \mathrm{~g}$ of substance, according to TLC-method; the original hydroxyethylaniline remained. 
The resulting mixture was separated on the silica gel column (Merck M60); the eluent was a mixture of ethyl acetate and petroleum ether (40-70) in a ratio of $1: 3$, followed by increase in content of polar component to 1: 1. The fractions with target product were combined and evaporated in vacuo. Received $2.2 \mathrm{~g}$ of chromophore 1 (yield 18\%) as a crystals with dark red color; m. p. $103-106{ }^{\circ} \mathrm{C}$.

${ }^{1} \mathrm{H}$ NMR (CDCl 3 , Bruker AM-300): $1.22(\mathrm{t}, 3 \mathrm{H}), 3.6(\mathrm{~m}, 2 \mathrm{H}), 3.85(\mathrm{t}, 4 \mathrm{H}), 6.8(\mathrm{~d}, 2 \mathrm{H}), 7.65(\mathrm{~d}, 2 \mathrm{H})$ 7.9 (m, 2H). ${ }^{13} \mathrm{C} \mathrm{NMR}\left(\mathrm{CDCl}_{3}\right):$ 11.58, 45.93, 52.31, 60.16, 106.09 (d), 111.54, 122 (q), 126.47, 129 (m), 143, 151.93, 156.78, 161 (d). ${ }^{19} \mathrm{~F} \mathrm{NMR}\left(\mathrm{CDCl}_{3}\right)$ : -56.97 (t, 3F), -111.02 (q, 2F). MS-ES $(70 \mathrm{eV}): \mathrm{M}+373$, calc. 373.33 .

\subsection{Synthesis of MMA-DR1218 ester}

The synthesis scheme for 4-(4-trifluoromethyl-3,5-difluorophenylazo) -N-ethyl-N- (2methacro-leyloxyethyl) aniline ester (MMA-DR1218) is shown in Fig. 7.

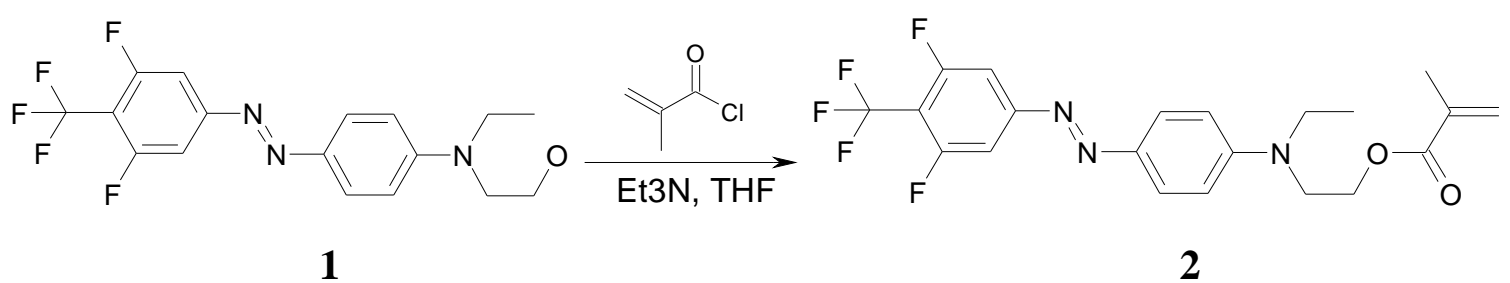

Figure 7. The synthesis scheme for 4-(4-trifluoromethyl-3,5-difluorophenylazo)-N-ethyl-N-(2methacroleoyloxyethyl) aniline ester. (1) - DR1218 chromophore, (2) - MMA-DR1218 ester.

4-(4-Trifluoromethyl-3,5-difluorophenylazo)-N-ethyl-N-(2-hydroxyethyl)aniline 1 (2.01 g, $5.4 \mathrm{mmol})$ was dissolved in $15 \mathrm{ml}$ of THF, triethylamine $(0.81 \mathrm{ml} 5.5 \mathrm{mmol})$ was added, cooled to 0 ${ }^{\circ} \mathrm{C}$ and the solution of $0.62 \mathrm{ml}(6.5 \mathrm{mmol})$ of freshly distilled methacryloyl chloride in $5 \mathrm{ml}$ of THF was added over 15 minutes with stirring via magnetic stirrer. It was stirred for 2 hours at $20{ }^{\circ} \mathrm{C}$; then another $0.81 \mathrm{ml}$ of triethylamine and $0.62 \mathrm{ml}$ of methacryloyl chloride were added and left overnight. This mixture was evaporated in vacuo via rotary evaporator, dissolved in $50 \mathrm{ml}$ of chloroform, washed with distilled water $(3 \times 20 \mathrm{ml})$ and dried over anhydrous $\mathrm{Na}_{2} \mathrm{SO}_{4}$. Sodium sulfate was filtered, the solution was passed through silica gel column (Merck 60) with $6 \mathrm{~cm}$ high and $3 \mathrm{~cm}$ in diameter; then this solution rotary evaporated to a constant weight at $65{ }^{\circ} \mathrm{C}$ to remove methacrylic acid impurities. $1.61 \mathrm{~g}$ (yield 67.3\%) of ester 2 were obtained as maroon oil, $\mathrm{R}_{\mathrm{f}} 0.5$ (Silufol, hexane-ethyl acetate 2:1).

${ }^{1} \mathrm{H}$ NMR spectrum $\left(\mathrm{CDCl}_{3}\right.$, Bruker AM-300): $1.28(\mathrm{t}, 3 \mathrm{H}), 1.95(\mathrm{~s}, 3 \mathrm{H}), 3.58(\mathrm{k}, 2 \mathrm{H}), 3.76(\mathrm{t}, 2 \mathrm{H})$, 4, $30(\mathrm{t}, 3 \mathrm{H}), 5.60(\mathrm{~s}, 1 \mathrm{H}), 6.13(\mathrm{~s}, 1 \mathrm{H}), 6.81(\mathrm{~d}, 2 \mathrm{H}), 7.47(\mathrm{~d}, 2 \mathrm{H}), 7.90(\mathrm{~d}, 2 \mathrm{H}))$. 
${ }^{19} \mathrm{~F}$ NMR Spectrum $\left(\mathrm{CDCl}_{3}\right): 57.07(3 \mathrm{~F}), 110.9(2 \mathrm{~F})$.

\subsection{Production of an electro-optical MMA-DR1218/FluoroAc polymer}

The scheme of EO-polymer MMA-DR1218/FluoroAc production by radical copolymerization of 4-(4-trifluoromethyl-3,5-difluorophenylazo)-N-ethyl-N-(2-methacroleyl-hydroxyethyl) aniline and 2,2,2-trifluoroethyl acrylate (FluoroAc) is shown in Fig. 8.

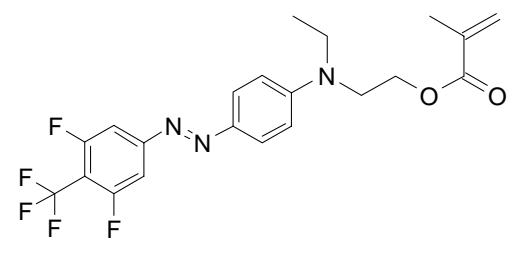

2

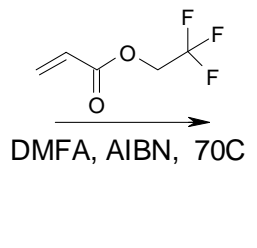

$$
\mathrm{F}^{\top}
$$

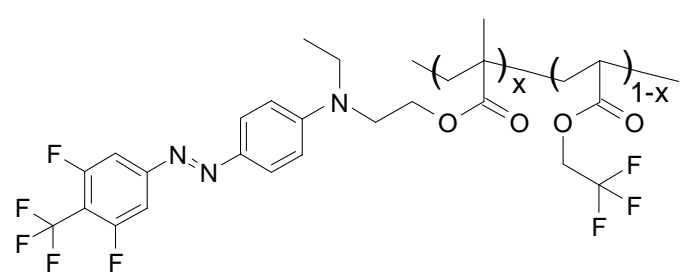

3

Figure 8. Scheme for producing polymer MMA-DR1218/FluoroAc 3. $x$-molar concentration of units with a covalently attached chromophore in macromolecule.

Compound 2 (2.29 g, $5.2 \mathrm{mmol})$ and 2,2,2-trifluoroethyl acrylate ( $800 \mathrm{mg}, 2.8 \mathrm{mmol})$ were dissolved in $5 \mathrm{ml}$ of freshly distilled DMF, $130 \mathrm{mg}$ of AIBN (3 mol\%) was added and stirred at 70 ${ }^{\circ} \mathrm{C}$ within 8 hours. After this, the mixture was cooled to $20{ }^{\circ} \mathrm{C}$; after another 16 hours $130 \mathrm{mg}$ of AIBN was added and resumed stirring at $70{ }^{\circ} \mathrm{C}$. This procedure was repeated again, after which the spot of substance 2 on TLC disappeared. The resulting mixture was poured into $100 \mathrm{ml}$ of methanol cooled to $0^{\circ} \mathrm{C}$; the polymer 3 precipitated as a red-orange viscous oil. This oil was evaporated to constant weight via rotary evaporator at $70{ }^{\circ} \mathrm{C}$, then it was dissolved in $100 \mathrm{ml}$ of chloroform and this solution was washed three times with $50 \mathrm{ml}$ of water each time; the chloroform solution was dried with $\mathrm{Na}_{2} \mathrm{SO}_{4}$ and evaporated. The resulting MMA-DR1218/FluoroAc copolymer 3 is soluble in dichloromethane, chloroform, chlorobenzene, DMF, limitedly soluble in methanol. To remove the low molecular weight fractions and purify from ionic impurities, the copolymer was dissolved in methanol and re-precipitated in water to isolate the target product. The obtained polymer $\mathbf{3}$ as a solid powder of dark red color, $1.82 \mathrm{~g}$, yield $58.2 \%$.

${ }^{1} \mathrm{H}$ NMR spectrum $\left(\mathrm{CHCl}_{3}\right.$, Bruker AM-300) of synthesized polymer is shown in Fig. 9. 


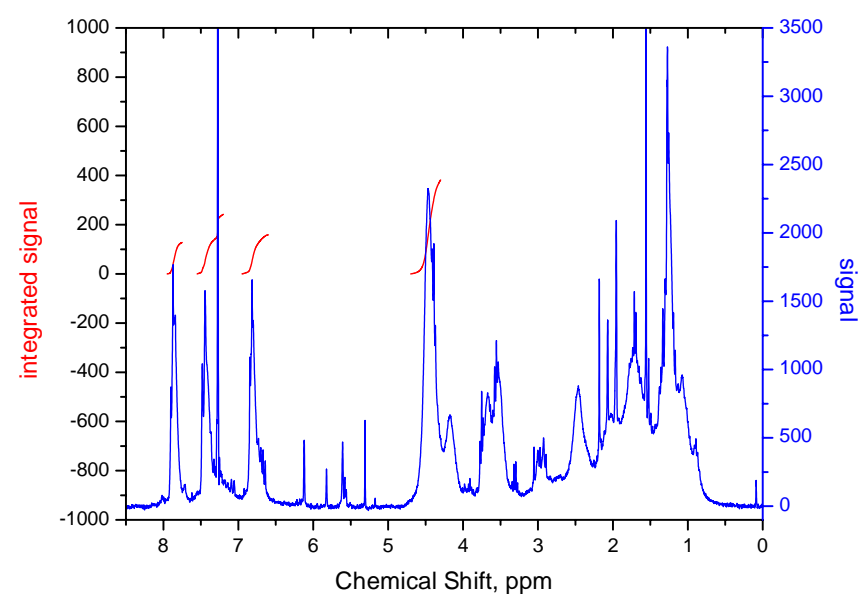

Figure 9. ${ }^{1} \mathrm{H}$ NMR spectrum of MMA-DR1218/FluoroAc copolymer with fluorine-containing electro-optical chromophore DR1218 in the side chain.

From the analysis of spectrum presented in Fig. 9, we can conclude that the ratio of links for 2,2,2-trifluoroethyl acrylate and chromophore methacrylic ester in polymer macromolecule is $\approx 3: 1$, which follows from a comparison of integral signal intensities at $7.8 \mathrm{ppm}$. (2 protons of benzene ring with fluorine substituents) and $4.5 \mathrm{ppm}$ (2 protons of $\mathrm{OCH}_{2} \mathrm{CF}_{3}$ group). Thus, the degree of fluorination of obtained polymer, defined as a ratio of fluorine atoms number to the number of fluorine and hydrogen atoms in macromolecule, is $28.6 \%$. The degree of fluorination can be increased, for example, by using, when copolymerizing, instead of 2,2,2-trifluoroethyl acrylate, the monomer 2,2,2-trifluoroethyl-1-fluoroacrylate $\mathrm{CH}_{2}=\mathrm{CF}-\mathrm{C}(\mathrm{O}) \mathrm{O}-\mathrm{CH}_{2} \mathrm{CF}_{3}$. The average molecular weight of obtained copolymer was $M_{\mathrm{w}}=4.8 \mathrm{kDa}$.

\section{Conclusion}

A new fluorine-containing electro-optical MMA-DR1218/FluoroAc polymer was synthesized with fluorine-containing chromophore covalently attached in the side chain. The degree of fluorination of polymer is $28.6 \%$, which ensures its high optical transparency in telecommunication C-band.

Light guide films are made from this polymer. It is shown that, under the action of laser radiation at wavelength of $440 \mathrm{~nm}$, the photobleaching of polymer occurs, accompanied by a decrease in the refractive index $n$ of material. The measured decrease in $n$ at wavelength of $632.8 \mathrm{~nm}$ was $\Delta n=0.04$, which allows the formation of various waveguide elements of integrated optical devices in this material under the action of laser radiation. 


\section{Literature}

1. Dalton. L., Benight S., Theory-Guided Design of Organic Electro-Optic Materials and Devices, Polymers, 2011, 3, 1325. 10.3390/polym3031325.

2. Liu J., Xu G., Liu F., Kityk I., Liu X., Zhen Z., Recent advances in polymer electro-optic modulators, RSC Advances, 2015, 5, 1578. 10.1039/C4RA13250E.

3. Zhang H., Oh M.C., Szep A., Steier W.H., Zhang C., Dalton L.R., Erlig H., Chang Y., Chang D.H., Fetterman H.R., Push-pull electro-optic polymer modulators with low half-wave voltage and low loss at both 1310 and 1550 nm, Applied Physics Letters, 2001, 78(20), 3136. 10.1063/1.1372203.

4. Zheng C.T., Zhang L.J., Qv L.C., Liang L., Ma C.S., Zhang D.M., Cui Z.C., Nanosecond polymer Mach-Zehnder interferometer electro-optic modulator using optimized micro-strip line electrode, Opt. Quant. Electron, 2013, 45(3), 279. 10.1007/s11082-012-9629-1.

5. Nazmieva G.N., Vakhonina T.A., Ivanova N.V., Mukhtarov A.Sh., Smirnov N.N., Yakimansky A.V., Balakina M.Yu., Sinyashin O.G., Testing of the ways for synthesis of new nonlinear optical epoxy-based polymers with azochromophores in the side chain, European Polymer Journal, 2015, 63, 207-216.

6. Sokolov V.I., Akhmanov A.S., Asharchuk I.M., Goryachuk I.O., Khaydukov K.V., Nazarov M.M., Formation of channel optical waveguides in polymethylmethacrylate with embedded electrooptic chromophore DR13 by the photoinduced bleaching method, Optics and Spectroscopy, 2017, 122(3), 128-134.

7. Michel S., Zyss J., Ledoux-Rak I., Nguyen C.T., High-performance electro-optic modulators realized with a commercial side-chain DR1-PMMA electro-optic copolymer, Proceedings of SPIE, Organic Photonic Materials and Devices XII, 2010, 7599, 75990I. 10.1117/12.841339.

8. V.I. Sokolov, A.S. Ahmanov, E.S. Vasilenko, I.O. Goriachuk, S.I. Molchanova, J. E. Pogodina, E.V. Polunin., Synthesis and investigation of optical properties of fluorine containing chromophore Disperse Orange DO1, Fluorine notes, 2018, 5(120), 1-2, 10.17677/fn20714807.2018.05.01.

9. Sokolov V.I., Ahmanov A.S., Asharchuk I.M., Goriachuk I.O., Zavarzin I.V., Pogodina J.E., Polunin E.V., Laser formation of light guides in electro optical polymers with fluorine containing chromophores in a side chain, FluorineNotes, 2018, 6(121), 5-6.

10. W. Groh, Overtone absorption in macromolecules for polymer optical fibers, Makromol. Chem., 1988, 189, 2861- 2874. 10.1002/macp.1988.021891213.

11. I. McCulloch, H. Yoon., Fluorinated NLO polymers with improved optical transparency in the Near Infrared., Journal of Polymer Science, Part A: Polymer Chemistry., 1995, 33, 1177-1183. 
12. Nakanishi M., Sugihara O., Okamoto N., Hirota K., Ultraviolet photobleaching process of azo dyedoped polymer and silica films for fabrication of nonlinear optical waveguides, Applied Optics., 1998, 37(6), 1068. 10.1364/AO.37.001068.

13. Denisyuk I.Y., Burunkova Y.E., Pozdnyakova S.A., Balya V.K., Zhuk D.I., Fokina M.I., An electro-optic polymer modulator for radio photonics, Optics and Spectroscopy, 2015, 119(4), 719-723. 10.7868/S0030403415100104. 